\title{
Comparison of risk-scoring systems in the prediction of outcome after liver resection
}

\author{
S. Ulyett ${ }^{1,2^{*}}$ D, G. Shahtahmassebi ${ }^{2,3}$, S. Aroori ${ }^{1}$, M. J. Bowles ${ }^{1}$, C. D. Briggs ${ }^{1}$, M. G. Wiggans ${ }^{1}$, G. Minto ${ }^{1,2}$ \\ and D. A. Stell ${ }^{1,2}$
}

\begin{abstract}
Background: Risk prediction techniques commonly used in liver surgery include the American Society of Anesthesiologists (ASA) grading, Charlson Comorbidity Index (CCI) and cardiopulmonary exercise tests (CPET). This study compares the utility of these techniques along with the number of segments resected as predictive tools in liver surgery.
\end{abstract}

Methods: A review of a unit database of patients undergoing liver resection between February 2008 and January 2015 was undertaken. Patient demographics, ASA, CCI and CPET variables were recorded along with resection size. Clavien-Dindo grade III-V complications were used as a composite outcome in analyses. Association between predictive variables and outcome was assessed by univariate and multivariate techniques.

Results: One hundred and seventy-two resections in 168 patients were identified. Grade III-V complications occurred after 42 (24.4\%) liver resections. In univariate analysis of CPET variables, ventilatory equivalents for $\mathrm{CO}_{2}$ $\left(\mathrm{VEqCO}_{2}\right)$ was associated with outcome. CCl score, but not ASA grade, was also associated with outcome. In multivariate analysis, the odds ratio of developing grade III-V complications for incremental increases in $\mathrm{VEqCO}_{2}$, $\mathrm{CCl}$ and number of liver segments resected were 1.09, 1.49 and 2.94, respectively.

Conclusions: Of the techniques evaluated, resection size provides the simplest and most discriminating predictor of significant complications following liver surgery.

Keywords: Preoperative assessment, Liver resection, Surgical complications, Cardiopulmonary exercise testing

\section{Background}

Despite the technical advances, liver resection remains potentially dangerous and is associated with a morbidity rate of $18.2-32.4 \%$ (Ulyett et al. 2015; Wiggans et al. 2014; Poon et al. 2004) and mortality rate of $1.4-5.3 \%$ (Nygard et al. 2012; Belghiti et al. 2000; Dimick et al. 2004). Preoperative estimation of risk allows counselling of patients regarding treatment options and helps in operative planning. A number of techniques are commonly used preoperatively to estimate risk including the American Society of Anesthesiology (ASA) grade, Charlson Comorbidity Index (CCI) and Cardiopulmonary Exercise Tests (CPET). The ASA grading system is a subjective assessment of the degree of systemic disease made at the time

\footnotetext{
* Correspondence: simon.ulyett@nhs.net

'Derriford Hospital, Plymouth PL6 8DH, UK

${ }^{2}$ Peninsula Schools of Medicine and Dentistry, Plymouth University, Plymouth PL6 8BU, UK

Full list of author information is available at the end of the article
}

of surgery (Saklad 1941). CCI is a 22-point scoring matrix based on comorbid diagnoses (Charlson et al. 1987), which was originally designed to predict long-term survival in an unselected population but has also been shown to be of value in predicting outcome after surgery (Backemar et al. 2015; Schmolders et al. 2015). CPET provides an objective measurement of cardiorespiratory fitness, where the volume of oxygen consumption at peak $\left(\mathrm{VO}_{2}\right.$ peak $)$ and at anaerobic threshold (AT), ventilatory efficiency in the elimination of carbon dioxide $\left(\mathrm{CO}_{2}\right)$ (VEeqCO $\left.\mathrm{CO}_{2}\right)$, heart rate (HR) and oxygen $\left(\mathrm{O}_{2}\right)$ pulse (a surrogate measure of cardiac output) are measured. This technique was initially used to predict mortality in patients undergoing a range of abdominal procedures (Older et al. 1993) and has been shown to be of value in predicting outcomes in patients undergoing pancreatic (Chandrabalan et al. 2013) and vascular (Thompson et al. 2011) surgery. 
Data on the use of these tools in the context of liver surgery is scarce. The ASA grade has been shown to influence the development of postoperative complications after liver resection (Belghiti et al. 2000), and CCI has been assessed in the prediction of short-term outcomes (Schroeder et al. 2006). Data on the use of CPET before liver surgery is conflicting, with one study showing a useful correlation with complications (Junejo et al. 2012) and another showing only minimal association (Dunne et al. 2014). None of the tools takes into account the extent of the proposed operation, and no comparison between the techniques has been undertaken.

The aim of this study is to determine the relative value of these risk prediction tools in patients undergoing liver surgery and also to assess their value compared to risk prediction based on the extent of the surgical procedure undertaken.

\section{Methods}

A review of a prospectively maintained database of all patients undergoing resection of parenchymal liver lesions between February 2008 and January 2015 was undertaken. Follow up was completed in June 2015. The primary endpoint was development of Clavien-Dindo (CD) grade III-V complications.

To reduce heterogeneity of the study population, patients undergoing synchronous bowel resection or surgery for obstructing lesions of the proximal hepatic duct or in the presence of liver cirrhosis were excluded, as surgery in these situations is associated with higher risk (Wiggans et al. 2014; Belghiti et al. 2000; Das et al. 2001). Liver resection was undertaken using Cavitron Ultrasonic Surgical Aspirator (CUSA). General anaesthetic was administered by specialist liver anaesthetists. Low central venous pressures (CVP) were maintained although invasive CVP monitoring was not undertaken. The extent of liver resection was described according to the Brisbane Classification (Pang 2002). Radiofrequency ablation (RFA) was used where major liver resections were performed leaving a residual contralateral disease. Where subsegmental resections were undertaken, these were rounded up to the nearest integer in analyses. Postoperatively, all patients undergoing major resection were cared for in a critical care unit. Retrieved data include age, gender, indication for surgery and use of preoperative chemotherapy. ASA grade was determined at the time of surgery by the responsible anaesthetist, and CCI was calculated postoperatively from clinical records. The use of perioperative blood transfusion was also recorded.

CPET was introduced as a preoperative assessment tool in February 2008 and was initially available at the discretion of referring consultants for patients considered to be at higher risk of surgical complications. After November
2013, CPET has been undertaken in the majority of patients. CPET was undertaken using a cycle ergometer (nSpire $^{\text {ma }}$ Zan $^{\circ}$ 600, Colorado, USA, or MGC Diagnostics $\left.{ }^{\circ}, \mathrm{MN}, \mathrm{USA}\right)$. The protocol consists of 2 mins of rest, 1 min of cycling without resistance, and then a ramped protocol of between 10 and 25 watts $/ \mathrm{min}$. CPET variables measured included $\mathrm{VO}_{2}$ peak, anaerobic threshold (AT), $\mathrm{O}_{2}$ pulse, relative $\mathrm{O}_{2}$ pulse, resting $(\mathrm{rHR})$ and peak heart rate $(\mathrm{pHR})$ and ventilatory equivalents for $\mathrm{CO}_{2}\left(\mathrm{VEqCO}_{2}\right)$. AT was calculated using the $\mathrm{V}$-slope method and $\mathrm{VO}_{2}$ peak was averaged over the last $30 \mathrm{~s}$ of the test. CPET were undertaken and interpreted by three specialist liver anaesthetists. In patients where AT was not achieved, a nominal low value of $8 \mathrm{ml} / \mathrm{kg} / \mathrm{min}$ was assigned as this group have been shown to have poor outcomes (Lai et al. 2013; Challand et al. 2012).

Postsurgical outcomes occurring within 30 days of surgery were classified according to the $\mathrm{CD}$ system (Dindo et al. 2004). Broadly, grade I-II complications include minor variations in the patient pathway including the use of anti-emetics and antibiotics, grade III complications require postoperative intervention (commonly for bile leaks), grade IV complications are determined by organ failure and grade $\mathrm{V}$ complication is death. In this study, grade III-V complications were used as a composite outcome of significant adverse postoperative events. Patients may develop complications in more than one grade, particularly as grade III and IV complications may have different aetiology. Liver failure was classified according to the International Study Group for Liver Surgery consensus definition of post-hepatectomy liver failure (PHLF) (Rahbari et al. 2011) and renal failure according to the RIFLE scoring system (Bellomo et al. 2004). For the purposes of this study, heart failure was defined as the requirement for inotropic medication to treat hypotension of suspected cardiac cause, after removal of epidural catheter. Respiratory failure was defined as a return to critical care unit for respiratory support.

Statistical analyses were carried out using chi-square and Mann-Whitney $U$ tests for categorical and continuous data, respectively. Binary logistic regression was used to assess the effect of risk factors on outcome. Repeat resections in individual patients were analysed separately where separate CPET were performed.

Confirmation was obtained from the South-West Health Research Authority that Research Ethics Committee review was not required because patient data were collected prospectively as a normal part of hospital care, and all data were anonymised. No patient consent was required for this study. This study was registered with the Research Registry (unique identification 464) (Research Registry) and conforms with the STROBE guidelines (von Elm et al. 2008). 


\section{Results}

Details of patients selected for the study are shown in Fig. 1. Nine (5\%) patients had repeat resections. Patient and operative characteristics, CPET parameters and ASA and CCI scores are shown in Table $1 . \mathrm{VEqCO}_{2}$ results were unavailable in five patients. Intraoperative RFA was used in addition to resection in 11 patients and portal vein embolization prior to resection in three patients. Laparoscopic resection was performed on 32 (18.6\%) occasions.

A surgeon estimated that the intraoperative blood loss was less than $500 \mathrm{ml}$ in 81 (47\%) and more than $1000 \mathrm{ml}$ in 31 (18\%) resections. Forty-four patients required a blood transfusion intraoperatively with a median transfusion of 2 units (1-18) for those transfused. Clavien-Dindo grade III to V complications occurred following 42 (24\%) of 172 resections (Table 2). Eleven patients suffered both III and IV/V complications. One patient developed heart failure secondary to cardiac arrhythmia requiring cardiac pacing. There were no cases of postoperative respiratory failure. The proportion of patients suffering grade III-V complications in the selective and non-selective CPET periods was similar (22 and $27 \%$, respectively).

Demographic details, ASA, CCI, use of pre-operative chemotherapy, CPET variables and the number of liver segments resected in patient groups with and without significant postoperative complications are shown in Table 3. In univariate analysis CCI score, but not ASA grade, was associated with grade III-V complications $(P=0.02)$. Of the CPET variables, $\mathrm{VEeqCO}_{2}$ was associated with the development of grade III-V complications $(P=0.005)$. AT was not detectable in three patients due to very limited exercise tolerance, and none of whom suffered grade III-V complications. The number of liver segments resected was strongly associated with outcome (Fig. 2). The use of preoperative chemotherapy was not associated with postoperative complications.

Multivariate analysis of predictive scores and the extent of liver resection shows that the extent of resection, $\mathrm{VEqCO}_{2}$ and $\mathrm{CCI}$ are independently associated with the development of grade III-V complications (Table 4). ASA grade was not associated with outcome. The strongest association with complications was shown to be with the extent of liver resection, where each extra segment of liver resected increased the odds of developing CD grade III-V complications by a factor of 2.94 (1.864.66). The greatest range of predictive values (17-50) was noted for $\mathrm{VEqCO}_{2}$, where an incremental increase of 1.09 was noted in the OR of developing grade III-V complications.

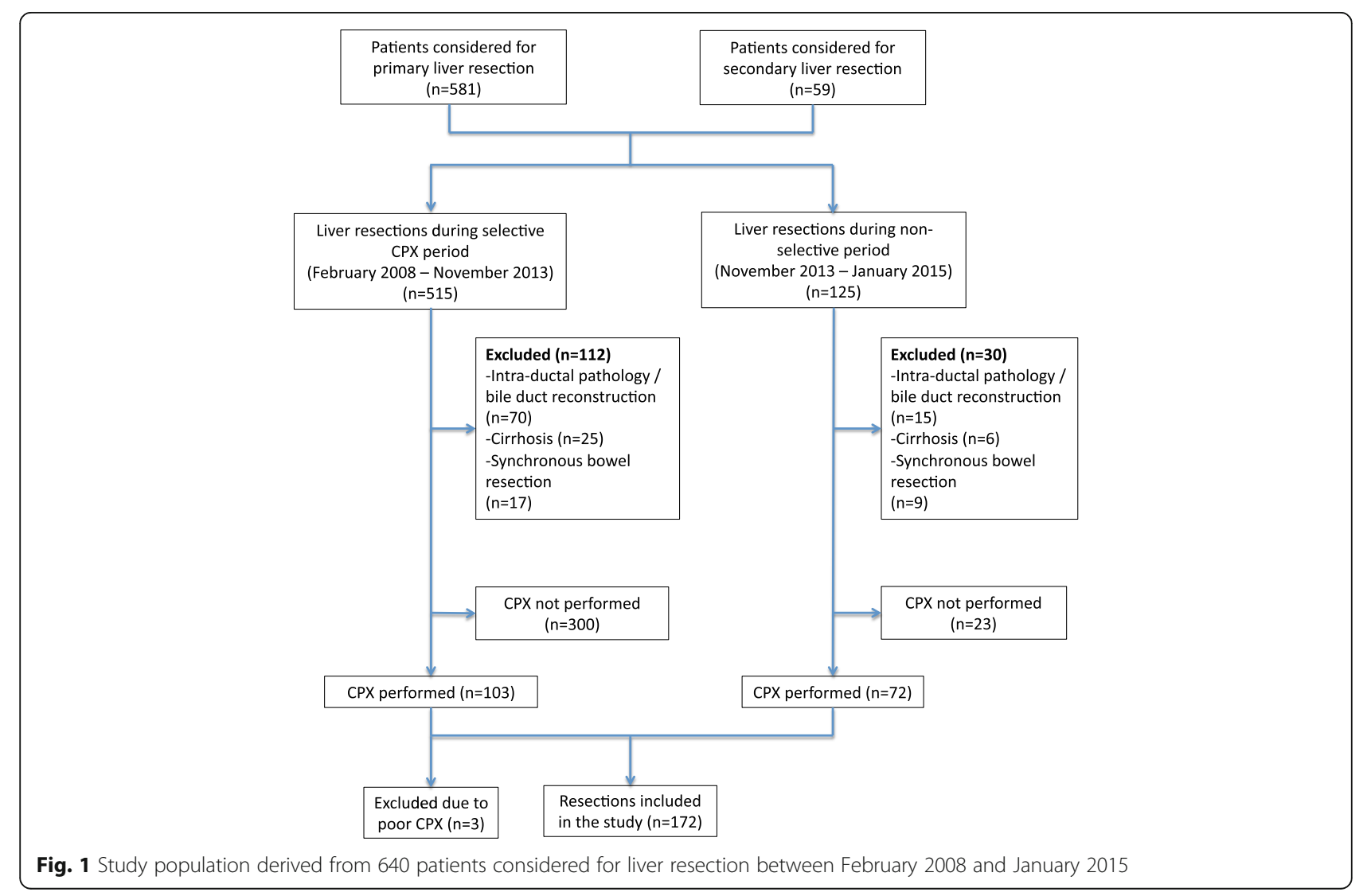


Table 1 Baseline patient characteristics

\begin{tabular}{|c|c|c|c|}
\hline Age (median [range]) & $69(22-90)$ & CPET parameters & \\
\hline \multicolumn{4}{|l|}{ Gender (\%) } \\
\hline Male & $119(69.2)$ & \multirow{2}{*}{$\begin{array}{l}\mathrm{VO}_{2} \text { at } \mathrm{AT} \\
(\mathrm{ml} / \mathrm{kg} / \mathrm{min} \text {, [range] })\end{array}$} & \multirow[t]{2}{*}{$12.5(5.6-23.1)$} \\
\hline Female & $53(30.8)$ & & \\
\hline \multicolumn{4}{|l|}{ Diagnosis (\%) } \\
\hline $\begin{array}{l}\text { Colorectal } \\
\text { metastases }\end{array}$ & $134(77.9)$ & $\begin{array}{l}\mathrm{VO}_{2 \text { peak }} \\
(\mathrm{ml} / \mathrm{kg} / \mathrm{min} \text {, [range]) }\end{array}$ & $18.9(6.4-35.5)$ \\
\hline Hepatoma & $12(7)$ & & $11.8(5-25.3)$ \\
\hline $\begin{array}{l}\text { Neuroendocrine } \\
\text { tumour }\end{array}$ & $6(3.5)$ & $\begin{array}{l}\text { Oxygen pulse } \\
\text { (ml/beat, [range]) }\end{array}$ & \\
\hline Other & $20(11.6)$ & & \\
\hline \multicolumn{4}{|l|}{ ASA (\%) } \\
\hline । & $8(4.7)$ & & $14.9(7.8-26.6)$ \\
\hline$\|$ & $93(54.1)$ & $\begin{array}{l}\text { Relative oxygen pulse } \\
(100 \mathrm{ml} / \text { beat/kg, [range]) }\end{array}$ & \\
\hline III & $70(40.7)$ & & \\
\hline IV & $1(0.6)$ & & \\
\hline CCl (median [range]) & $4(0-9)$ & $\begin{array}{l}\text { Resting heart rate } \\
\text { (beats/min, [range]) }\end{array}$ & $77(39-119)$ \\
\hline $\begin{array}{l}\text { Preoperative } \\
\text { chemotherapy (\%) }\end{array}$ & & $\begin{array}{l}\text { Peak heart rate } \\
\text { (beats/min, [range]) }\end{array}$ & $132(79-180)$ \\
\hline Yes & $62(36)$ & & $29.6(17.1-49.9)$ \\
\hline No & $94(54.7)$ & & \\
\hline Unknown & $16(9.3)$ & $\begin{array}{l}\text { VEeqCO } 2 \text { at AT } \\
\text { (range) }\end{array}$ & \\
\hline \multicolumn{4}{|l|}{ RFA used (\%) } \\
\hline Yes & $11(6.4)$ & & \\
\hline No & $161(93.6)$ & & \\
\hline \multicolumn{4}{|c|}{ Number of segments resected (\%) } \\
\hline 1 & $46(26.7)$ & & \\
\hline 2 & $13(7.6)$ & & \\
\hline 3 & $14(8.1)$ & & \\
\hline 4 & $64(37.2)$ & & \\
\hline 5 & $31(18)$ & & \\
\hline 6 & $4(2.3)$ & & \\
\hline
\end{tabular}

Demographic and operative characteristics of 172 liver resections in 168 patients who had preoperative CPET

$R F A$ radiofrequency ablation

\section{Discussion}

The main finding of this study is the very significant association between postoperative complications in liver surgery and the extent of the liver resection undertaken. There is a weaker association between postoperative complications and risk scores formulated by assessment of recorded comorbidity (CCI) and CPET parameters. Subjective assessment of a patient's fitness at the time of surgery by ASA score is not predictive of postoperative complications.

The scoring systems under assessment in this study were chosen because they assess risk by different techniques. CPET is an objective measure of cardiovascular fitness,
Table 2 Summary of complications after liver resection

\begin{tabular}{lc}
\hline Grade III complications (\%) & $15(8.6)$ \\
- Bile leak requiring ERCP & $3(1.7)$ \\
- Bile leak requiring drain & $4(2.3)$ \\
- Infected collection requiring drain & $1(0.6)$ \\
• Further surgery required & $2(1.1)$ \\
• Open drainage of collection following colonic injury, & \\
• Laparotomy undertaken for ileus & \\
- Pneumothorax requiring drain & $1(0.6)$ \\
- Cardiac pacing & $1(0.6)$ \\
- Ascites requiring drain & $1(0.6)$ \\
- Pleural effusion requiring drain & $1(0.6)$ \\
- Gastric burn secondary to radiofrequency ablation & $1(0.6)$ \\
Grade IV and V complications (\%) & $37(21.3)$ \\
- Liver failure alone & $24(13.8)$ \\
- Renal failure alone & $6(3.4)$ \\
- Liver and renal failure & $6(3.4)$ \\
• Heart failure & $2(1.1)$ \\
- Death & $2(1.1)$ \\
\hline Summary of Clavien-Dindo grade III-V postoperative complications following \\
172 liver resections in 168 patients. Some patients suffered more than \\
one complication
\end{tabular}

CCI scores are derived from recorded comorbidity, ASA grade is a subjective assessment of overall health and the number of liver segments resected provides a simple measure of operative extent. Assessment of the techniques in parallel allows a comparison of their relative utility.

The role of CPET in liver surgery is yet to be established, with conflicting outcomes from the two published studies investigating the technique (Junejo et al. 2012; Dunne et al. 2014). CPET is useful in surgery where cardiorespiratory complications form a major part of adverse outcomes, such as vascular (Thompson et al. 2011; Elkouri et al. 2004) and cardiothoracic (Van Diepen et al. 2014) surgery. As the main cause of morbidity and death after liver surgery is liver failure (Poon et al. 2004; Belghiti et al. 2000; Jarnagin et al. 2002), the degree of cardiovascular fitness is likely to have a weaker association with outcome. In our series, significant cardiovascular complications were rare, occurring in only two patients. Also, patients undergoing liver resection may be a selected group with better physical function, as many will have previously undergone, and recovered from, primary colorectal surgery. AT was originally shown to be useful in predicting mortality in a large, unselected population undergoing a range of elective abdominal procedures including vascular surgery, in which $24 \%$ of patients had evidence of preexisting cardiovascular disease (Older et al. 1993), and mortality in this context is more likely to be related to cardiovascular health. 
Table 3 Comparison of potential risk factors for developing complications

\begin{tabular}{|c|c|c|c|}
\hline Variable (median) & CD 0-\| $(n=130)$ & $C D \| I-V(n=42)$ & $P$ value \\
\hline Age (range) & $69(22-90)$ & 70 (49-88) & 0.47 \\
\hline Gender (\%) & & & 0.057 \\
\hline Male & $85(65.4)$ & $34(81)$ & \\
\hline Female & $45(34.6)$ & $8(19)$ & \\
\hline ASA (\%) & & & 0.187 \\
\hline$|-| \mid$ & $80(61.5)$ & $21(50)$ & \\
\hline III-IV & $50(38.5)$ & $21(50)$ & \\
\hline $\mathrm{CCl}$ (range) & $4(0-7)$ & $5(1-9)$ & 0.021 \\
\hline Preoperative chemotherapy (\%) & & & 0.85 \\
\hline Yes & $46(35.4)$ & $16(38.1)$ & \\
\hline No & $71(54.6)$ & $23(54.8)$ & \\
\hline Unknown & $13(10)$ & $3(7.1)$ & \\
\hline Number of segments resected (range) & $3(1-6)$ & $4(3-6)$ & $<0.001$ \\
\hline $\mathrm{VO}_{2}$ at $\mathrm{AT}(\mathrm{ml} / \mathrm{kg} / \mathrm{min}$, [range]) & $12.8(6.4-22.9)$ & $12.5(5.6-23.1)$ & 0.84 \\
\hline $\mathrm{VO}_{2 \text { peak }}(\mathrm{ml} / \mathrm{kg} / \mathrm{min}$, [range] $)$ & $18.8(6.4-35.5)$ & $19.2(11.8-30.8)$ & 0.65 \\
\hline Oxygen pulse (ml/beat, [range]) & $11(5-20.5)$ & $12.3(5-25.3)$ & 0.39 \\
\hline Relative oxygen pulse (100 ml/beat/kg, [range]) & $15(7.8-26.6)$ & $14.9(10-24.5)$ & 0.52 \\
\hline Resting heart rate (beats/min, [range]) & $74(39-115)$ & $80(43-119)$ & 0.54 \\
\hline Peak heart rate (beats/min, [range]) & $131.5(79-180)$ & $133.5(85-172)$ & 0.89 \\
\hline$V_{\text {EeqCO }}$ at AT (range) & $29.1(17.1-49.9)$ & $31.7(24.4-46.2)$ & 0.005 \\
\hline
\end{tabular}

Univariate analysis of association of age, gender, diagnosis, ASA grade, CCl, preoperative chemotherapy, extent of resection and CPET values with Clavien-Dindo $0-$ II and III-V complications following 172 liver resections

Of the CPET parameters under study, $\mathrm{VEqCO}_{2}$ was shown to be predictive of postoperative complications, in keeping with earlier findings (Junejo et al. 2012). Although the incremental OR for predicting CD grade III-V complications is low (1.09), this effect is noted over a large range of values (17-50). Despite other studies demonstrating the value of AT in the prediction of outcomes after liver resection (Dunne et al. 2014), this parameter was not shown to be of value in this series, although many of the CPET variables are mathematically related, and the difference in predictive value between them may be less than is apparent in a multivariate analysis. In a similar manner to $\mathrm{CCI}$, the median $\mathrm{VEqCO}_{2}$ was very similar between groups with CD grade $0-\mathrm{II}$ and III-V complications (29.1 vs 31.7) with significant overlap in range, and this may limit the usefulness of this test. ASA was not shown to

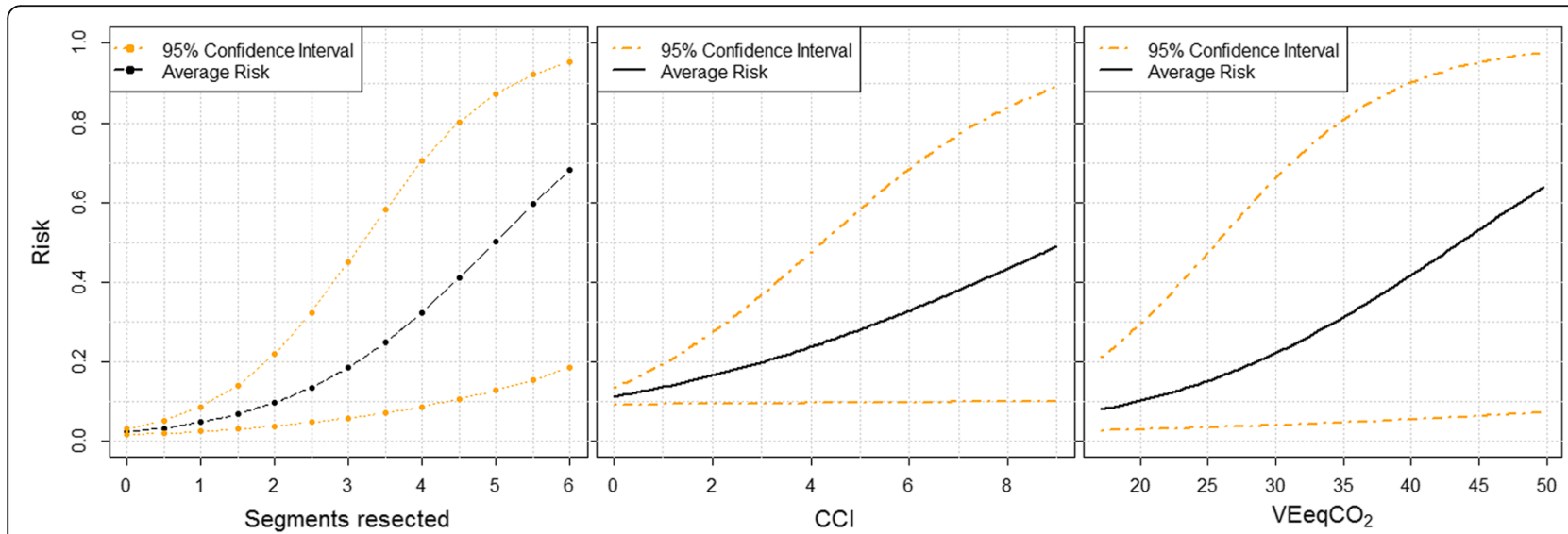

Fig. 2 Association of a extent of liver resection, $\mathbf{b} \mathrm{CCl}$ score and $\mathbf{c} \mathrm{VEqCO}_{2}$ with the risk of developing Clavien-Dindo grade III-V complications following 172 liver resections. N.B. The number of liver segments resected was analysed as a discrete variable, whereas the $\mathrm{CCl}_{\text {and }} \mathrm{VEqCO}$ were analysed as continuous variables 
Table 4 Comparison of risk assessment techniques

\begin{tabular}{lllll}
\hline & OR & $95 \% \mathrm{Cl}$ & $P$ value & $\begin{array}{l}\text { Range of } \\
\text { predictive } \\
\text { values }\end{array}$ \\
\hline ASA grade & 0.85 & $0.37-1.94$ & 0.7 & $1-4$ \\
$\mathrm{CCl}$ score & 1.49 & $1.09-2.04$ & 0.01 & $0-9$ \\
VEeqCO 2 at AT & 1.09 & $1.01-1.17$ & 0.04 & $17-50$ \\
$\begin{array}{l}\text { Number of liver } \\
\text { segments resected }\end{array}$ & 2.94 & $1.86-4.66$ & $<0.001$ & $1-6$
\end{tabular}

Multivariate analysis of risk assessment techniques in the prediction of $C D$ grade III-V complications compared with grade 0 -II complications following 172 liver resections

predict outcome in multivariate analysis compared with the other measures. ASA is known to be a highly subjective tool, with significant inter-observer variation (Mak et al. 2002; Ranta et al. 1997). In practice, this is also too blunt a tool to be of value, as $95 \%$ of patients have ASA score of II or III.

The CCI is a well-researched measure used to weight outcomes in cancer surgery (Dobbins et al. 2015). The tool has also been used in registry data when comparing outcomes between individual hospitals (Dobbins et al. 2015) and clinicians (Ugolini and Nobilio 2004). Its role in predicting specific surgical complications is variable. It has not been shown to be associated with complications in gynaecologic or colorectal surgery (Suidan et al. 2015; Krarup et al. 2015), but is associated with outcome in orthopaedic surgery (Schmolders et al. 2015). While $\mathrm{CCI}$ is associated with complications in this study, it is less predictive of outcome over the range of measured values than either the number of liver segments resected or $\mathrm{VEqCO}_{2}$ (Fig. 2). Although CCI provides a simple measure that can be easily calculated with knowledge of a patient's medical history, the median score of patients suffering grade III-V complications is only one point higher than those with grade $0-$ II complications, with a large overlap in the score range which limits the usefulness of the technique. Also, the CCI system records comorbidity rarely relevant in the context of elective liver surgery, including AIDS and lymphoma, while other more common potential risk factors, such as extremes of BMI (Vigano et al. 2011; Balzan et al. 2010) and NAFLD (Wakai et al. 2011), are not included.

The factor with the greatest predictive value for outcome in this analysis is the extent of the liver resection undertaken, which may be expected as liver failure due to insufficient liver volume is the major cause of death after liver surgery (Wiggans et al. 2013). The high odds ratio of 2.94 in the prediction of grade III-V complications for increasing number of resected segments make this a factor of high clinical relevance and easily utilised, as the extent of liver resection to be undertaken is usually known preoperatively. Of note, the increased risk of liver resection with increasing number of resected segments is non- linear, with the largest absolute increase in risk being experienced by patients undergoing resection of five and six liver segments.

Each of the three techniques shown to be of value has very wide confidence intervals in the prediction of outcome (Fig. 2). The potential risk faced by patients undergoing liver surgery is affected by all of the factors under study (comorbidity, physical functioning and extent of liver resection), and each of the tests used individually therefore will be limited in their predictive value. Other factors are also likely to influence operative risk, particularly the presence of a coexisting liver disease. A useful area of further study would be to develop a compound risk scoring system based on all these factors.

The study may be subject to bias due to patient selection. Throughout the series, CPET has been undertaken on patients perceived to be less healthy, which accounts for the male predominance of the population under study. It is unlikely however that CPET parameters would be more predictive of complications in a healthier population. A potential limitation of this study is the degree of confounding caused by prior awareness of CPET results by clinicians, as it is possible that patients with low levels of fitness were treated differently. Preoperative medication which may affect CPET results has not been recorded. However, in undertaking CPET, it is important to estimate functional parameters as they would be at the time of surgery, including the influence of medication.

\section{Conclusions}

This study represents a large case-series, which attempts to answer the question of how best to assess risk in patients undergoing liver resection. The simplest factor to consider is the extent of the planned liver resection. CCI and CPET parameters may be useful discriminators for potential risk in patients undergoing the same resection type and may also contribute to decision-making in patients who require extended liver resections.

\section{Abbreviations \\ AIDS: Acquired immunodeficiency syndrome; ASA: American Society of Anesthesiology; AT: Anaerobic threshold; BMI: Body mass index; CCl: Charlson Comorbidity Index; CD: Clavien-Dindo; CPET: Cardiopulmonary Exercise Testing; HR: Heart rate; NAFLD: Non-alcoholic fatty liver disease; $\mathrm{O}_{2}$ : Oxygen; PHLF: Post-hepatectomy liver failure; RFA: Radiofrequency ablation; VEeqCO $\mathrm{C}_{2}$ : Ventilatory efficiency in the elimination of carbon dioxide; $\mathrm{VO}_{2}$ peak: Volume of oxygen consumption at peak}

\section{Acknowledgements}

None.

Funding

None.

Availability of data and materials

Data is securely held within Plymouth NHS Trust. Use of this data would need to be approved by Plymouth NHS Trust. 


\section{Authors' contributions}

$\mathrm{SU}$ is responsible for the study design, data collection and interpretation and manuscript preparation. GS contributed to the data interpretation, production of Fig. 2 and review of the statistical methods. SA, MJB, CDB and MGW took part in the data collection. GM did the setup of the CPET service and helped with the CPET data collection and interpretation. DAS contributed to the study design, data collection and interpretation and manuscript preparation.

All authors read and approved the final manuscript.

\section{Ethics approval and consent to participate}

Confirmation was obtained from the South-West Health Research Authority that Research Ethics Committee review was not required because patient data were collected prospectively as a normal part of hospital care, and all data were anonymised.

\section{Consent for publication}

Not applicable.

\section{Competing interests}

The authors declare that they have no competing interests.

\section{Publisher's Note}

Springer Nature remains neutral with regard to jurisdictional claims in published maps and institutional affiliations.

\section{Author details}

'Derriford Hospital, Plymouth PL6 8DH, UK. ${ }^{2}$ Peninsula Schools of Medicine and Dentistry, Plymouth University, Plymouth PL6 8BU, UK. ${ }^{3}$ Nottingham Trent University, Nottingham NG1 4BU, UK.

\section{Received: 3 November 2016 Accepted: 16 October 2017}

Published online: 25 November 2017

\section{References}

Backemar $\mathrm{L}$, et al. Comorbidities and risk of complications after surgery for esophageal cancer: a Nationwide cohort study in Sweden. World J Surg. 2015;39:2282-8.

Balzan S, et al. Safety of liver resections in obese and overweight patients. World J Surg. 2010;34:2960-8.

Belghiti J, et al. Seven hundred forty-seven hepatectomies in the 1990s: an update to evaluate the actual risk of liver resection. J Am Coll Surg. 2000;191:38-46.

Bellomo R, et al. Acute renal failure: the second international consensus conference of the acute dialysis quality initiative (ADQI) group. Crit Care. 2004:8:R204-12.

Challand C, et al. Randomized controlled trial of intraoperative goal-directed fluid therapy in aerobically fit and unfit patients having major colorectal surgery. Br J Anaesth. 2012;108:53-62.

Chandrabalan W, et al. Pre-operative cardiopulmonary exercise testing predicts adverse post-operative events and non-progression to adjuvant therapy after major pancreatic surgery. HPB (Oxford). 2013;15:899-907.

Charlson ME, et al. A new method of classifying prognostic comorbidity in longitudinal studies: development and validation. J Chronic Dis. 1987;40:373-83.

Das BC, et al. Analysis of 100 consecutive hepatectomies: risk factors in patients with liver cirrhosis or obstructive jaundice. World J Surg. 2001;25:266-72. discussion 72-3

Dimick JB, et al. National trends in the use and outcomes of hepatic resection. J Am Coll Surg. 2004:199:31-8.

Dindo D, et al. Classification of surgical complications: a new proposal with evaluation in a cohort of 6336 patients and results of a survey. Ann Surg. 2004;240:205-13.

Dobbins TA, et al. Assessing measures of comorbidity and functional status for risk adjustment to compare hospital performance for colorectal cancer surgery: a retrospective data-linkage study. BMC Med Inform Decis Mak. 2015;15:55.

Dunne DF, et al. Cardiopulmonary exercise testing before liver surgery. J Surg Oncol. 2014;110:439-44.

Elkouri S, et al. Perioperative complications and early outcome after endovascular and open surgical repair of abdominal aortic aneurysms. J Vasc Surg. 2004; 39:497-505.
Jarnagin WR, et al. Improvement in perioperative outcome after hepatic resection: analysis of 1,803 consecutive cases over the past decade. Ann Surg. 2002;236:397-406. discussion -7

Junejo MA, et al. Cardiopulmonary exercise testing for preoperative risk assessment before hepatic resection. Br J Surg. 2012;99:1097-104.

Krarup PM, et al. Association of Comorbidity with Anastomotic leak, 30-day mortality, and length of stay in elective surgery for colonic cancer: a Nationwide cohort study. Dis Colon rectum. 2015;58:668-76.

Lai CW, et al. Patients' inability to perform a preoperative cardiopulmonary exercise test or demonstrate an anaerobic threshold is associated with inferior outcomes after major colorectal surgery. Br J Anaesth. 2013;111:607-11.

Mak PH, et al. The ASA physical status classification: inter-observer consistency. American Society of Anesthesiologists. Anaesth Intensive Care. 2002;30:633-40.

Nygard IE, et al. Mortality and survival rates after elective hepatic surgery in a low-volume centre are comparable to those of high-volume centres. ISRN Surgery. 2012;2012:783932.

Older $\mathrm{P}$, et al. Preoperative evaluation of cardiac failure and ischemia in elderly patients by cardiopulmonary exercise testing. Chest. 1993;104:701-4.

Pang YY. The Brisbane 2000 terminology of liver anatomy and resections. HPB 2000; 2:333-39. HPB (Oxford). 2002:4(2):99.

Poon RT, et al. Improving perioperative outcome expands the role of hepatectomy in management of benign and malignant hepatobiliary diseases: analysis of 1222 consecutive patients from a prospective database. Ann Surg. 2004:240:698-708.

Rahbari NN, et al. Posthepatectomy liver failure: a definition and grading by the international study Group of Liver Surgery (ISGLS). Surgery. 2011;149:713-24.

Ranta S, et al. A survey of the ASA physical status classification: significant variation in allocation among Finnish anaesthesiologists. Acta Anaesthesiol Scand. 1997:41:629-32.

Research Registry. editor^, editors " City. http://www.researchregistry.com/.

Saklad M. Grading of patients for surgical procedures. Anesthesiology. 1941;2: $281-4$.

Schmolders J, et al. Validation of the Charlson comorbidity index in patients undergoing revision total hip arthroplasty. Int Orthop. 2015;39:1771-7.

Schroeder RA, et al. Predictive indices of morbidity and mortality after liver resection. Ann Surg. 2006;243:373-9.

Suidan RS, et al. Predictive value of the age-adjusted Charlson Comorbidity index on perioperative complications and survival in patients undergoing primary debulking surgery for advanced epithelial ovarian cancer. Gynecol Oncol. 2015;138:246-51.

Thompson AR, et al. Cardiopulmonary exercise testing provides a predictive tool for early and late outcomes in abdominal aortic aneurysm patients. Ann R Coll Surg Engl. 2011;93:474-81.

Ugolini C, Nobilio L. Risk adjustment for coronary artery bypass graft surgery: an administrative approach versus EuroSCORE. Int I Qual Health Care. 2004;16:157-64

Ulyett $\mathrm{S}$, et al. Clinical assessment prior to hepatectomy identifies high-risk patients. J Surg Res. 2015;198:87-92.

Van Diepen S, et al. Predicting cardiovascular intensive care unit readmission after cardiac surgery: derivation and validation of the Alberta provincial project for outcomes assessment in coronary heart disease (APPROACH) cardiovascular intensive care unit clinical prediction model from a registry cohort of 10,799 surgical cases. Crit Care. 2014;18:651.

Vigano L, et al. Liver resection in obese patients: results of a case-control study. HPB (Oxford). 2011;13:103-11.

von Elm E, et al. The strengthening the reporting of observational studies in epidemiology (STROBE) statement: guidelines for reporting observational studies. J Clin Epidemiol. 2008:61:344-9.

Wakai T, et al. Surgical outcomes for hepatocellular carcinoma in nonalcoholic fatty liver disease. J. Gastrointest. Surg. 2011;15:1450-8.

Wiggans MG, et al. Renal dysfunction is an independent risk factor for mortality after liver resection and the main determinant of outcome in posthepatectomy liver failure. HPB Surg. 2013;2013:875367.

Wiggans MG, et al. The interaction between diabetes, body mass index, hepatic Steatosis, and risk of liver resection: insulin dependent diabetes is the greatest risk for major complications. HPB Surg. 2014;2014:586159. 